I. A. Lami and H. S. Maghdid, "Synchronising WiFi access points with GPS time obtained from smartphones to aid localisation," presented at the International Conference on Computer Applications Technology (ICCAT), Sousse, Tunisia, 2013.

(C) 2013 IEEE. Personal use of this material is permitted. Permission from IEEE must be obtained for all other uses, in any current or future media, including reprinting/republishing this material for advertising or promotional purposes, creating new collective works, for resale or redistribution to servers or lists, or reuse of any copyrighted component of this work in other works. 


\section{Synchronising WiFi Access Points with GPS time obtained from Smartphones to aid localisation}

\author{
Ihsan Alshahib Lami \\ Applied Computing Department \\ The University of Buckingham \\ Buckingham MK18 1EG, UK \\ ihsan.lami@buckingham.ac.uk
}

\author{
Halgurd S. Maghdid \\ Applied Computing Department \\ The University of Buckingham \\ Buckingham MK18 1EG, UK \\ halgurd.maghdid@buckingham.ac.uk
}

\begin{abstract}
WiFi Access Points (WAPs) are readily available everywhere for access to the Internet. Unless specifically designed for localisation applications and their position accurately calibrated, WAPs do not offer accurate position calculation and do not know their geographic position. However, Smartphones, with the aid of onboard GPS and WiFi will know their location to within few meters when outdoors. This paper proposes to utilise Smartphones, while outdoors, to sync the clock-time and define the location of any WAP within reach. This is achieved by using Trilateration techniques where the Smartphones become reference stations at several instances in time as they approach available WAPs. Also, any WAP position is continuously updated as other Smartphones approach them. Such WAPs can later be used as reference stations to aid indoors localisation. Simulation scenarios based on MATLAB implementation has proved the viability of this scheme.
\end{abstract}

Keywords - WiFi Access Point Localisation; Clock Synchronisation; GPS time synchronisation

\section{INTRODUCTION}

WAPs are used to connect to the Internet in most buildings/homes, for example from PCs, Tablets and Smartphones. Some location-based services (LBS) applications on Smartphones use WAPs pre-defined location (that was stored on a database/Server) to fix the approximate location of the Smartphones [1]. LBS growth is predicted to grow by $13 \%$ year on year reaching $€ 200$ Billion in 2016 [2]. This is due to many wireless technologies available onboard Smartphones, such as GPS, Cellular, and Bluetooth that increased the breadth and complexity of applications [3].

Current Smartphone LBS, using GPS and/or network-based localisation technologies, are based on Trilateration techniques. Smartphones can achieve reliable accuracy of its location to within few meters when outdoors [4]. However, for indoors localisation, solutions are normally tailor-made with specialised hardware because of multipath/loss of GPS signals. If WAPs have accurate reference time, accurate clocks as well as pre-defined accurate position, then Smartphones can use WAPs as reference stations to locate them indoors. However, due to costs and function limitations, WAPs include a lowgrade clock and cannot locate themselves.

Our proposal does not require dedicated hardware (host server, sensors, and calibration) that are typically associated with indoors solutions such as Fingerprinting [5]. Therefore, our scheme shall reduce the required memory and traffic on Smartphones thus saving battery consumption, connection/interaction traffic and processing time. Additionally, the WAPs time-synchronisation shall be based on GPS-time. This means that our proposed "indoors localisation scheme" can be performed seamlessly, anywhere, anytime, with any Smartphone i.e., the proposed scheme includes two novelties: (1) synchronising all "WAPs clock time" with GPS time, on the Smartphone. (2) Consequently, locating WAPs via Smartphones, based on the Time-Of-Arrival (TOA) technique.

Our Scheme starts when a Smartphone is outdoors and has already acquired a GPS fix. As this Smartphone approaches buildings, it shall receive WAPs beacon frames, and will measure their pseudoranges. Thus by knowing its instantaneous geographical location and the WAPs pseudoranges, this Smartphone can compute these WAPs locations. Pseudoranges measurement is based on the WAPs GPS time synchronisation. After which, the various located and synchronised WAPs can act as reference points for calculating the Smartphones location indoors, especially when GPS signals disappears. Thus, when deployed at large scale via several Smartphones and WAPs, such scheme can offer seamless outdoors-indoors localisation. The freshly calculated WAP location can also be transported into a central-server-database on the fly from any participating Smartphone, thus saving the costs and time involved in using purpose built vehicles to survey WAPs location [1].

During implementation and trials of our scheme, we found out that implementation of the proposed scheme on actual Android based Smartphones and using off-the-shelf WAPs has many challenges; including access to these WiFi transceiver and GPS receivers Firmware. Therefore, we have initially focused on proving the scheme based on MATLAB simulation. Also, to achieve accurate simulation results, a WAP clock model was required; therefore, an accurate and dynamic WAP clock time model has been developed [6].

Section II elaborates on other work in this area and highlights the advantages of our proposed scheme. Section III details the proposed synchronisation/localisation algorithms in this scheme, while Section IV explains the scenarios, implementation and discusses the simulation results to prove the proposed scheme. Finally, Section V concludes the 
achievements of this work and highlights future work planned for this scheme.

\section{RELATED WORK}

WiFi technology can be used as an alternative way for defining Smartphones location, especially in environments when GNSS signals do not exist such as indoors and/or urban areas [7]. To achieve this, accurate WAPs location information must be available on the go, as and when needed from any WAP in any vicinity, and without prior special arrangements. For example, in existing WiFi positioning techniques such as Fingerprinting and Signal Propagation Law techniques [5].

Two localisation schemes have been developed by Skyhook and Ekahau offer WAP based localisation solutions. These schemes use the Fingerprint-technique. Purpose built vehicles is deployed to conduct a full WAPs' coordinate survey in builtup areas. When a user wants to locate his/her Smartphone, using Skyhook [1] for example, the localisation application will scan nearby WAPs for MAC address, and then sends the collected addresses to a Skyhook's server via an Internet connection. Then, the server calculates the rough position based on the nearest WAP location stored on its database and sends back the location of the Smartphone to the application. This solution incurs large cost to surveying the WAPs in that location.

A laptop hosted system including a GPS receiver used to define reference points as well as an external motorised-WiFi antenna is proposed to detect the direction of the WAP's signal at two different points based on the signals' Angle-Of-Arrived (AOA) technique by calculating the maximum Received Signal Strength (RSS) value to localise neighbouring WAPs. After obtaining WAPs location information, this system displays the location co-ordinates on a Google Earth application on the host laptop [8]. However, the position measurements have large errors due to fluctuating WiFi signal strength (this fluctuation varies based on the path of the received packet all the time). To implement this system, Smartphones will have to include extra hardware such as the motorised antenna to detect the WiFi signal direction, which is not practical.

Another RSS-based measurement technique to calculate the WAP pseudoranges from a GPS enabled device has been published recently [9]. This is similar to our proposal in that it treats the Smartphone, located by the onboard GPS receiver, as a reference station to help locate WAPs. However, this technique is based on pseudoranges measurement with heuristic algorithms to assure the WAP position. Again, since the strength of any WAP signal is not stable and its fluctuation is dependent on the WiFi protocol used, the simulation results published showed that, when outdoors, the WAPs are located within 5 meter error with probability of about $80 \%$. That means that the accuracy will be worse indoors. Also, carrying out such heuristic calculations on a Smartphone will be too much power drain on its battery. An enhanced attempt was proposed by establishing a relationship between RSS and pseudoranges of WAPs from Smartphones, where a linear regression is applied to estimate linear coefficients that are used to perform Trilateration [10]. This solution works even if there is no information (e.g. path loss exponent and transmission power) available on the WAPs signals. However, the geometric relationship between the WAP and RSS measurements, for example, where the geometric conditions are poor (worst case), the estimated coefficients would be variable and the position error becomes relatively large.

Our study has concluded that WAPs signals strength has a non-uniform shadowing and so any pseudorange estimation based on RSS would not be accurate. Also, reflections, and multipath affect RSS based localisation accuracy. Thus, to mitigate such errors, some computational mechanisms are needed such as the local signal strength gradient algorithm [11]. This algorithm claims to improve RSS-positioning technique accuracy. The idea of the gradient algorithm is to find the direction of the received signal by pointing arrows towards the WAPs. However, this algorithm is based on using heuristic or estimated values, such as estimating a window size for any measurement, and this will vary in different locations. Also, if the window size is too small, the result of the direction estimation is likely to have a huge error.

Our proposed scheme does away with RSS by locating WAPs via Smartphones, when outdoors, using the TOAtechnique that has been proven to offer more accurate location [12]. Also, GPS time is used to assure the accuracy of TOA by having both the WAP and Smartphone sync to the same time. Finally, our scheme does not require any extra hardware added/used for performing this localisation. As a by product, a central server can be deployed, similar to Skyhook server, to store the location information of WAPs in a dynamic way as they get updated by nearby Smartphones, instead of using dedicated signal-survey vehicles.

\section{OUR SYNCHRONISATION/LOCALISATION SCHEME}

The first novelty of this scheme is to use GPS-time obtained by nearby Smartphones when outdoors to synchronise WAPs clock, instead of using any other reference time (e.g. Network Time). Direct or Indirect clock synchronisations are possible: 1) Direct clock synchronisation is achieved any WAP within the vicinity of the Smartphone will sync its clock directly with the Smartphone's GPS receiver clock. WAPs' beacon signals are used to achieve the synchronisation with Smartphone. Since the clocks of the WAPs are continuous on their skew, see Fig 1a, a virtual WAPs clock time synchronisation can be done by calculating WAPs clocks' skew and storing them in the Smartphone's memory. These are then used to compensate the WAPs clock error during the localisation.
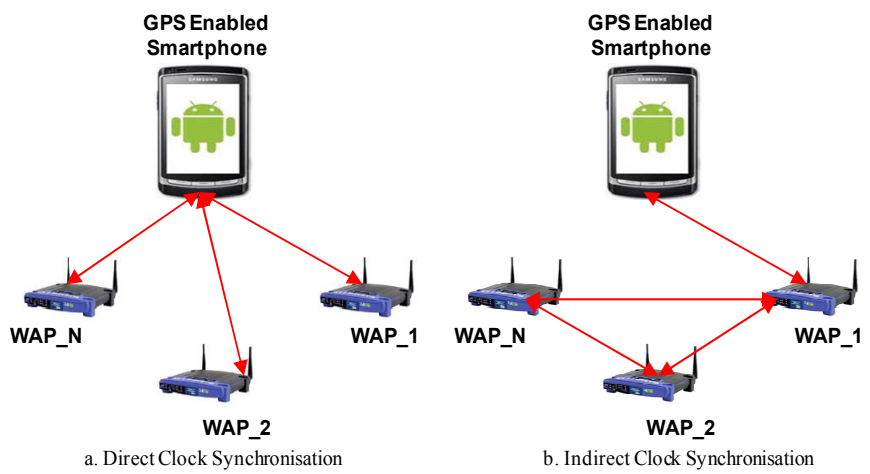

Figure 1. Proposed clock synchronisation algorithms 
2) Indirect clock synchronisation is achieved when a nearby WAP sync its clock with the Smartphone's GPS receiver clock, and then pass-on this time to other WAPs on its wired network, as shown in Fig 1b. This method is useful when the Smartphone can only receive a single WAP beacon signal, at the location of the synchronisation. For both methods, the WiFi transceiver firmware needs to be modified on the Smartphones, while the indirect method also necessitates a firmware modification to all WAPs on the network.

The second novelty is to establish the location of these synchronised WAPs via Smartphones based on the TOA technique. When a GPS-enabled Smartphone (or more) is outdoors travelling in the vicinity of any WAP, it can receive the WAP's beacon signals. Then, this Smartphone can measure its pseudoranges with this WAP by knowing the timestamp of the transmitted and received beacon signals. Of course, measuring the pseudoranges is based on the clock synchronisation that has been already established based on GPS-time. Thus, estimated pseudoranges will be very precise due to having accurate WAPs clock offset and clock drift. This Smartphone can then be considered as a reference station at that instance in time, and together with other Smartphones in the vicinity, the WAP position can be calculated based on TOA. If there are no other Smartphones available in the vicinity at that time, then the movement of this single Smartphone will be used to provide several reference points to enable coarse WAP position calculation. Fig. 2 illustrates this process where the number of reference points of the Smartphone movement can be predefined as a threshold.

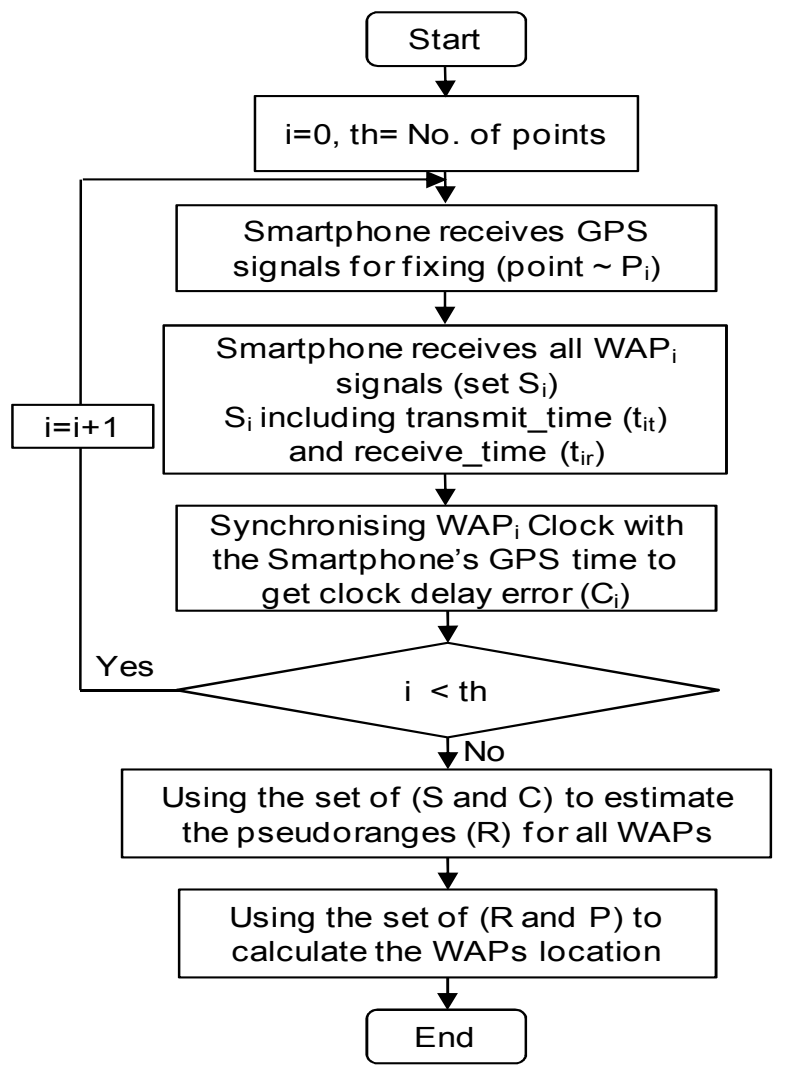

Figure 2. Flow chart for the scheme of synchronising/locating WAP via a Smartphone
In addition, the Smartphone(s) compares defined WAPs position all the time to ascertain an accurate position for these WAPs. These WAPs positions can also be transmitted wirelessly to be store in an Internet hosted central serverdatabase. Also, these localised and time-synchronised WAPs can be used as reference stations to aid localisation of the Smartphones when indoors; thus offering seamless outdoorsindoors Smartphone localisation anywhere anytime.

\section{TEST SCENARIOS, SIMULATION AND RESULTS}

To prove our proposed scheme, Android-based Smartphones are selected because Android is fully supported, widely used and proved, open and free mobile platform. Specifically, for WiFi time-based localisation, Android Kernel supports several time functions such as the Kernel timers, CPU clock and WiFi MAC clock. However, these time functions resolution and accuracy are at a higher level to that required for localisation. This is because (1) the Kernel timers have huge time degradation (time drift/delay) since they are interrupted by "software interrupts", as shown in Fig. 3, (2) the CPU clock is more accurate than the Kernel timers due to being handled by "hardware interrupts" only, but this is still in microsecond accuracy (a 1 microsecond time error is equal to 300 meters of position error), (3) the accuracy of WiFi MAC clock is $\sim 22.5$ nanosecond, which is equivalent to approximately 6.75 meter of positioning error. Therefore, all these time functions are not accurate for synchronising WAPs clock or for position calculations. This is why we chose GPS time which is based on the atomic clock.

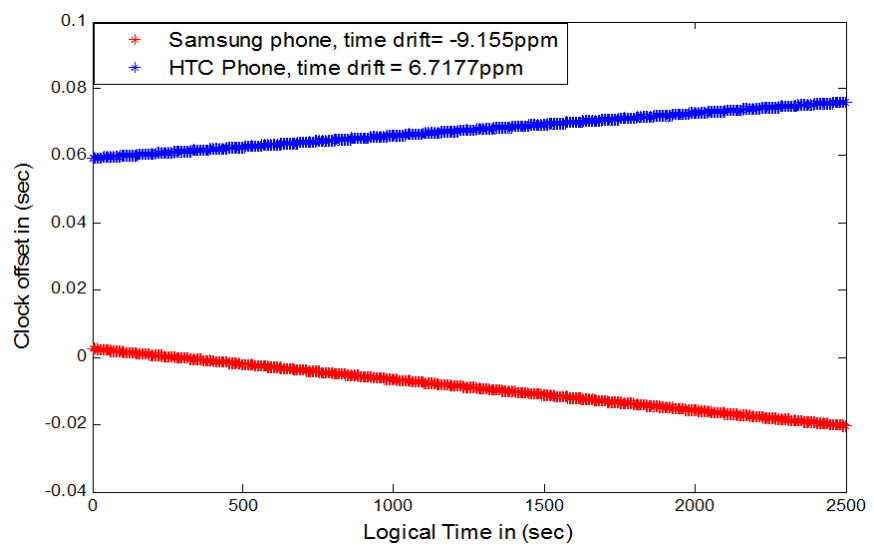

Figure 3. Example of Android-based Smartphones time degradation

GPS receivers, onboard Smartphones, provide the GPS time via NMEA messages every second with a resolution in millisecond. To achieve a time resolution in nanosecond necessary for accurate positioning, we therefore should modify the Smartphone GPS receiver firmware to output nanosecond resolution time available from the GPS signal. For our experiments, we are planning to use an external GPS receiver that outputs nanoseconds GPS time such as the Garmin GPS receivers [13].

Changing/modifying the firmware of the onboard Smartphone GPS receiver and $\mathrm{WiFi}$ transceiver for implementing our proposed scheme on actual Android based Smartphones are the main challenges that we need to resolve at 
the moment. Therefore, initially, this scheme is proved by simulation in MATLAB.

\section{A. Test Scenarios/Hypotheses}

A single and 4-Smartphone scenarios are tested in MATLAB as shown in Fig. $4 \mathrm{a}$ and Fig. $4 \mathrm{~b}$ respectively. Single Smartphone WAP positioning has very high Dilution of Precision (DOP) due to the reference points will be near each other. Therefore, multi-Smartphone positioning will be much more accurate due to a lower DOP value (DOP value in TOAtechnique depends on geometric condition of the reference stations and the target station). Also, to test both scenarios in different geographical anticipation, four hypotheses are conducted as follows.

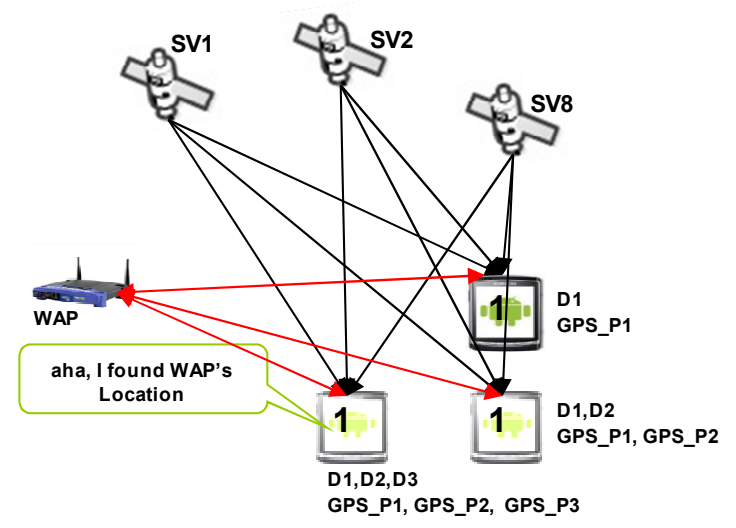

a. Single-Smartphone scenario

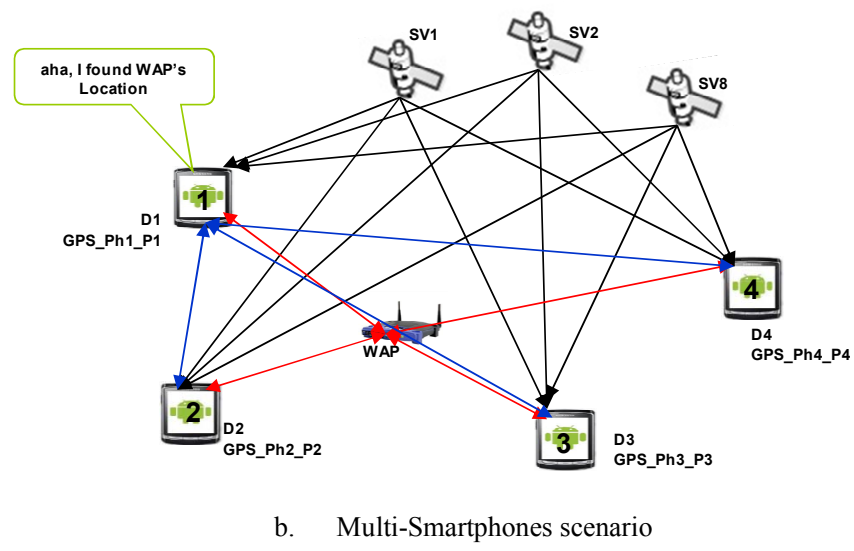

Figure 4. Locating WAPs Scenarios via Smartphone(s)

In the first hypothesis, we assumed that a Smartphone is located in three continuous different points, and at the same time the Smartphone can synchronise the WAP clock and measures its pseudoranges (in this work; we choose the direct clock synchronisation algorithm). Thus, the Smartphone can calculate the WAP's position in a single iteration.

In the second hypothesis, the Smartphone has updated GPS position fixed in seven continuous points and it has measured the WAP's pseudoranges at each point too. From the first three points, the Smartphone can calculate the WAPs position, and then at the next points the Smartphone updates the WAP's position information by the most recent calculation.

The third and fourth hypotheses are the same as the first and second hypothesis respectively except that the WAP time is re-synchronised with the Smartphone's GPS clock every 10 seconds.

All these 4 hypotheses are repeated for the 4-Smartphones scenario, but the Smartphones update the defined WAP's position at every point.

\section{B. Simulations Setup}

We chose an experiment area of $100 \mathrm{~m} \mathrm{X} 100 \mathrm{~m}$ to simulate our scenarios. Fig. 5 and Fig. 6 show the simulated test-bed experiments for the first and second scenarios, respectively. Assumed, there are four WAPs (green spots); their signals are available for a Smartphone regardless of multipath/loss signals. Also, the single Smartphone scenario start to end route travel around the area is shown by the yellow spots. While in the second scenario, the four Smartphones start/finish routes travel are at different places as shown by the yellow spots with the black, blue, red and pink lines respectively.

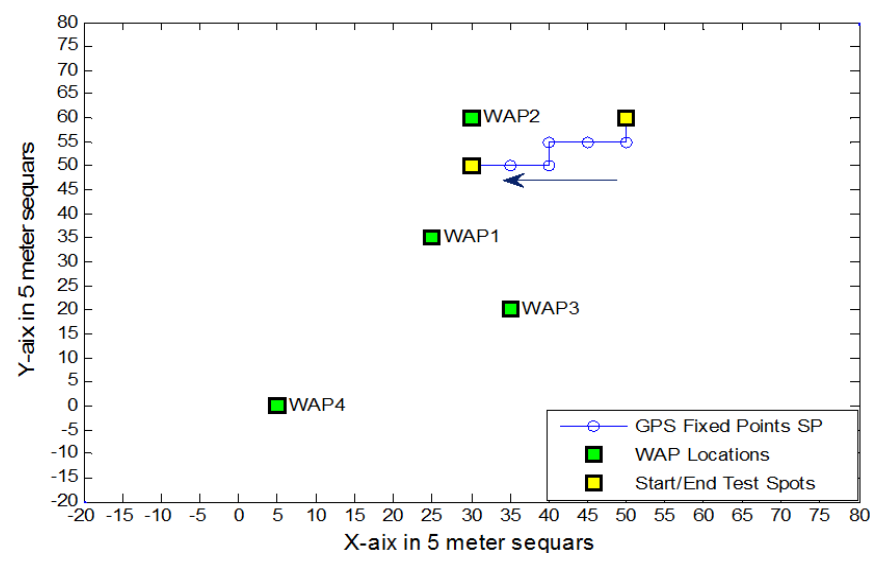

Figure 5. Simulated test-bed for the single-Smartphone Scenario

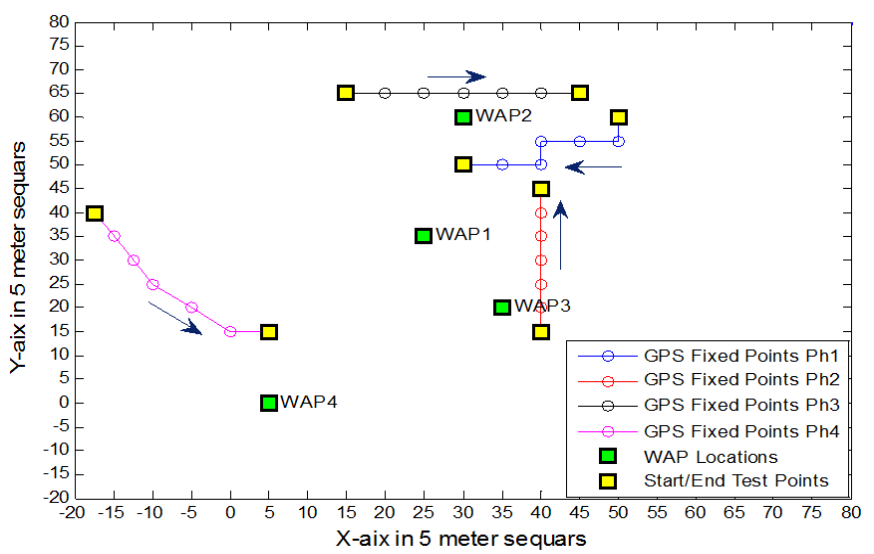

Figure 6. Simulated test-bed for the multi-Smartphones scenario 


\section{WAP Clock Model}

To simulate the WAP localisation scenarios, an accurate WAP clock model is needed in MATLAB (TOA technique is used). Therefore, we developed a new WAP clock time model for $\mathrm{WiFi}$ localisation applications to accurately measure the WAP clock offset and clock drift [6], i.e., the clock delay error is based on real clock noise sources of the WAP clock and using GPS time as a reference time. The clock model for generating WAP clock time can be computed by using equation (1).

$$
C_{\text {time }}=T+t^{*} C_{\text {offset }}+C_{\text {drift }}
$$

Where $\mathrm{C}_{\text {time }}$ is the local time of a WAP clock, $\mathrm{T}$ is the time offset according to reference time (e.g. GPS time); $t$ is a logical time (counter time increased in $1 \mathrm{~ms}$ ). $\mathrm{C}_{\text {offset }}$ is a clock offset in part per-million (ppm) and $\mathrm{C}_{\mathrm{drift}}$ is a drift of the clock model.

In both scenarios, the clock model is used to generate all WAPs clock time. To prove the novelty of using GPS-time as an accurate reference time, a simulated network time (Nettime) is used. The simulated Net-time is represented by synchronising all WAPs clock with a single/reference WAP clock in the network. So, both GPS-time and Net-time are used as a reference time to synchronise WAPs clock time separately. This WAPs clock time is used to stamp the generated beacon frames. Also, we assumed that the WAPs clock offset, in all cases, have a static value. That is, each WAPs clock delay error includes only clock drift.

Fig. 7 shows the WAP clock delay error generated for a single WAP, when the WAP clock is synchronised based on GPS time and the Net-time. It can be seen, when GPS-time is used to synchronise the WAP clock, the WAP clock delay error is more stable. Similarly, Fig. 8 shows the same, but resynchronised at every 10 seconds for the simulation of the third and fourth hypotheses. Note that re-synchronising or refreshing WAPs clock periodically has mitigated the effectiveness of the WAPs clock delay error on this localisation scheme.

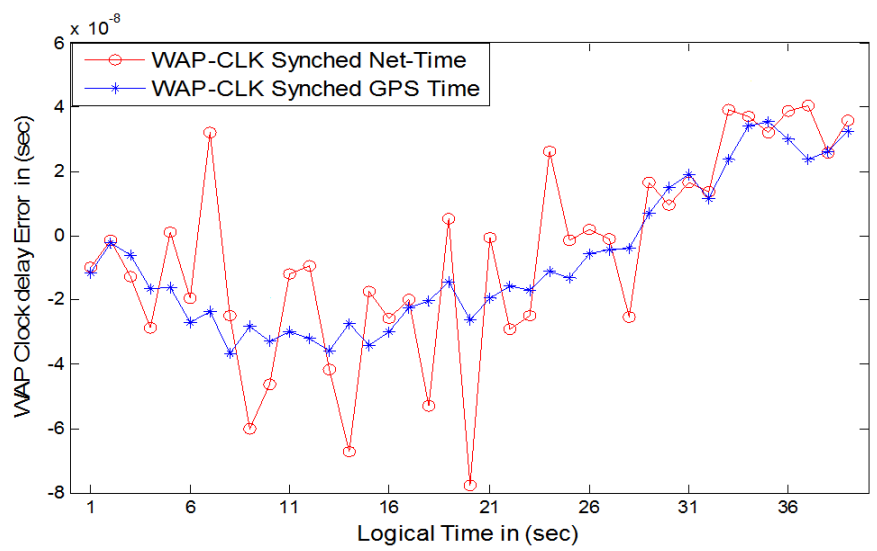

Figure 7. Generated a single WAP clock delay error based on GPS time and Net-time

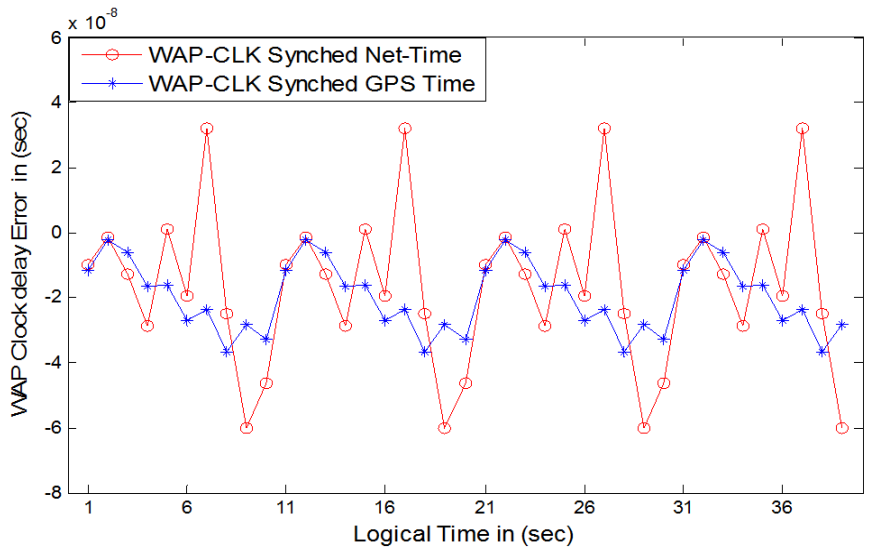

Figure 8. Generated a single WAP clock delay error based on GPS time and Net-time, and resynchronised at every 10 seconds

\section{Evaluation of Positioning Accuracy}

Table (I) shows the results of the WAP location accuracy through running all the hypotheses in both scenarios. The first hypothesis accuracy is worst, while the best accuracy is achieved in the fourth hypothesis. The results of the simulated scheme show that the multi-Smartphone scenario is more accurate than the single-Smartphone scenario. This is due to having poor geometric condition in the single-Smartphone scenario. Also, the best accuracy achieved was 0.9 meter using GPS-time, while it is 3.93 meter if the synchronisation is based on Net-time. Thus, better accuracy of WAPs location can be obtained when the GPS-Time is used as reference time for the clock synchronisation, in all cases. Also, the results show that, if the WAPs clock is re-synchronised every 10 seconds, then the accuracy is improved in both scenarios.

TABle I. Results of WAP LOCALISATION ACCURACY IN DifFERENT SCENARIOS/HYPOTHESES

\begin{tabular}{|c|c|c|c|c|c|}
\hline \multirow{2}{*}{ Scenarios } & \multirow{2}{*}{ Hypotheses } & \multicolumn{2}{|c|}{$\begin{array}{c}\text { Mean of WAP } \\
\text { location Accuracy in } \\
\text { meter }\end{array}$} & \multicolumn{2}{|c|}{$\begin{array}{l}\text { STD of WAP location } \\
\text { Accuracy in meter }\end{array}$} \\
\hline & & $\begin{array}{l}\text { Synched } \\
\text { Based on } \\
\text { GPS Time }\end{array}$ & $\begin{array}{l}\text { Synched } \\
\text { Based on } \\
\text { Net Time }\end{array}$ & $\begin{array}{l}\text { Synched } \\
\text { Based on } \\
\text { GPS Time }\end{array}$ & $\begin{array}{l}\text { Synched } \\
\text { Based on } \\
\text { Net Time }\end{array}$ \\
\hline \multirow{4}{*}{$\begin{array}{l}\text { Single- } \\
\text { Phone }\end{array}$} & Hypothesis 1 & 12.301 & 24.047 & 5.208 & 17.189 \\
\hline & Hypothesis2 & 12.536 & 17.838 & 4.609 & 14.960 \\
\hline & Hypothesis 3 & 6.3 & 16.058 & 6.592 & 8.793 \\
\hline & Hypothesis 4 & 3.985 & 9.368 & 4.295 & 6.009 \\
\hline \multirow{4}{*}{$\begin{array}{l}\text { Multi- } \\
\text { Phones }\end{array}$} & Hypothesis 1 & 11.862 & 18.958 & 4.203 & 12.610 \\
\hline & Hypothesis2 & 10.367 & 16.053 & 5.309 & 6.744 \\
\hline & Hypothesis 3 & 2.806 & 8.691 & 2.641 & 4.552 \\
\hline & Hypothesis4 & 0.918 & 3.930 & 0.898 & 2.167 \\
\hline
\end{tabular}

\section{CONCLUSION AND FUTURE WORK}

Locating WAPs by Smartphones is achievable. Storing such information in Smartphones' memory or on an Internet central-database can make such localisation system fullyautomated. This is especially useful when Smartphones can use this information to locate themselves indoors. This paper presents simulated experiments proving the viability of such scheme based on the TOA technique. 
The results of the simulation show that the important parameter to help achieve good positioning accuracy is the quality of the WAPs clock synchronisation. Also, to obtain high quality of clock synchronisation, an accurate reference time (like GPS time) is needed. Furthermore, the accuracy of WAP location by implementing multi-Smartphone scheme will be much more accurate than a scheme based on a singleSmartphone scenario. Also, Continuous or periodical synchronising of WAPs clock, i.e. getting refresh WAPs clock offset and clock drift, can improve localisation accuracy. Our simulation for the fourth hypothesis showed an accuracy of the WAPs location within one meter.

Thus, our scheme is a good candidate for seamless LBS localisation applications on Smartphones and without dependency on pre-installed and calibrated infrastructure or dedicated Internet based data/server.

Overcoming the difficulties of implementing this proposed scheme on the actual Android-based Smartphones will be the focus of this research work.

\section{ACKNOWLEDGMENT}

This research was sponsored by Koya University, Iraq.

\section{REFERENCES}

[1] T. Gallagher, B. Li, A. Kealy and A. Dempster, "Trials of commercial Wi-Fi positioning systems for indoor and urban canyons," in IGNSS 2009 Symposium on GPS/GNSS, 2009.

[2] E. G. Agency, "GNSS Market Report issue 2," 2012.

[3] E. Oliver, "A survey of platforms for mobile networks research," ACM SIGMOBILE Mobile Computing and Communications Review, vol. 12, no. 4, pp. 56-63, 2009.
[4] TruePosition, "U-TDOA Enabling New Location-based Safety and Security Solutions," White Paper, USA, October 2008.

[5] N. Kodippili and D. Dias, "Integration of fingerprinting and trilateration techniques for improved indoor localization," in Wireless And Optical Communications Networks (WOCN), 2010 Seventh International Conference On, 2010.

[6] H. Maghdid and I. Lami, "Dynamic Clock-Model of Wi-Fi AccessPoints to help Indoors Localisation of Smartphones," in IV International Congress on Ultra Modern Telecommunications and Control Systems ICUMT2012, St. Petersburg, 2012.

[7] B. Li, I. Quader and A. Dempster, "On outdoor positioning with Wi-Fi," Journal of Global Positioning Systems, vol. 7, no. 1, pp. 18-26, 2008.

[8] A. Ibrahim and D. Ibrahim, "Real-time GPS based outdoor WiFi localization system with map display," Advances in Engineering Software, vol. 41, no. 9, pp. 1080-1086, 2010.

[9] S. Chun, S. Lee, J. Nah, J. Choi and J. Park, "Localization of Wi-Fi Access Point using smartphone's GPS information," in Mobile and Wireless Networking (iCOST), 2011 International Conference on Selected Topics in, 2011.

[10] J. Koo and H. Cha, "Localizing WiFi access points using signal strength," Communications Letters, IEEE, vol. 15, no. 2, pp. 187-189, 2011.

[11] D. Han, D. Andersen, M. Kaminsky, K. Papagiannaki and S. Seshan, "Access point localization using local signal strength gradient," Passive and Active Network Measurement, pp. 99-108, 2009.

[12] S. Koenig, M. Schmidt and C. Hoene, "Multipath mitigation for indoor localization based on IEEE 802.11 time-of-flight measurements," in World of Wireless, Mobile and Multimedia Networks (WoWMoM), 2011 IEEE International Symposium, 2011.

[13] G. I. Inc., "GPS 18 Technical Specifications," 2005. 